\title{
IMPLEMENTACIÓN DE UN PROGRAMA EDUCATIVO, DIRIGIDO AL PERSONAL DE SALUD RESPONSABLE DEL CONTROL PRENATAL, ORIENTADO A PROMOVER LA SALUD ORAL DE LAS GESTANTES *
}

${ }^{1}$ Claudia Milena Riveros Alejo, ${ }^{2}$ Liliana Rocío Hormiga Parra, ${ }^{2}$ Tatiana Sofía Núñez Sarmiento, ${ }^{2}$ Nohora Camila Rugeles Páez. ${ }^{1}$ Odontóloga U. El Bosque, Especialista en Gerencia Hospitalaria, Escuela Superior de Administración Pública. Especialista en Relaciones Laborales, U. Autónoma de Bucaramanga. Especialista en Odontopediatría, U. Santo Tomás, Docente U. Santo Tomás. ${ }^{2}$ Estudiante $\mathrm{X}$ semestre F. de Odontología U. Santo Tomás.

Autor responsable de la correspondencia: Claudia Milena Riveros A.

Correo electrónico: milevale_odontopediatria@yahoo.es

\section{RESUMEN}

Objetivo: Implementar un programa educativo, dirigido al personal de salud responsable del control prenatal, orientado a promover la salud oral de las mujeres gestantes.

Materiales y métodos: Se realizó un ensayo clínico no controlado. Se eligieron 26 profesionales del programa de control prenatal y 40 gestantes que cumplían con los criterios de inclusión. Cada grupo se dividió en experimental y control. Los cuatro grupos se evaluaron mediante cuestionarios de conocimientos en prevención y salud oral en gestantes, antes y después de implementar un programa educativo. En las gestantes además de la encuesta se realizó un examen clínico, se valoraron los índices COP, CPITN y Cuantificado de Placa antes y después de la intervención. Se analizaron las variables evaluadas en los grupos de profesionales y de gestantes intervenidos y de control. Resultados: En relación con los conocimientos de los profesionales de la salud, se observaron diferencias estadísticamente significativas entre los grupos $(\mathrm{p}=0,030)$, ya que el porcentaje de respuestas acertadas antes de la intervención fue del 53,8\% que se incrementó después de la intervención $(84,6 \%)(p=0,013)$. En cuanto a las gestantes, se evidenciaron diferencias estadísticamente significativas en las variables $\operatorname{COP}(p=0,0342)$, CPITN $(p=0,000)$ y el índice cuantificado de placa $(p=0,0016)$.

Conclusión: La efectividad de un programa educativo dirigido a profesionales encargados del control prenatal puede mejorar las condiciones de salud oral en las gestantes. [Riveros CM, Hormiga LR, Nuñez TS, Rugeles NC. Implementación de un programa educativo, dirigido al personal de salud responsable del control prenatal, orientado a promover la salud oral de las gestantes. Ustasalud 2010; 9: 34 - 40]

Palabras clave: Odontología, Gestación, Educación oral, Salud oral, Salud pública.

\section{IMPLEMENTATION OF AN EDUCATIVE PROGRAM DIRECTED TO THE PERSONNEL OF HEALTH RESPONSIBLE FOR THE PRENATAL CONTROL, ORIENTED TO PROMOTE THE ORAL HEALTH OF THE PREGNANT ONES}

\begin{abstract}
Objective: Implementation of an educative material oriented to promote the oral health in pregnant women managed by the personnel of health responsible for the prenatal control.

Methods: A not controlled clinical test was done. There were chosen 26 professionals of prenatal control programs and 40 pregnant women that fulfilled the established criteria of inclusion. Each one of groups was divided in two, experimental and control. The four groups were evaluated by knowledge questionnaires about prevention and oral health in pregnant women before and after receiving an educative material. In the pregnant women also a clinical examination, DMFT, CIPTN, ICP and a Quantified index of Plaque exams were done before and after the intervention. There were analyzed the variables evaluated in the groups of professionals and pregnant women in the experimental and control groups.

Results: In relation with the knowledge on prevention in health's professionals, there were observed statistically significant differences $(p=0,030)$ between the groups because the average of $53,8 \%$ of positives answers before of intervention, increased, after the intervention $(84,6 \%)(p=0,013)$ between the groups. On the other hand, pregnant women showed statistically significant differences in the variables: DMFT $(p=0,0342)$, CPITN $(p=0,000)$, and the quantified index of plaque $(p=0,0016)$.

Conclusion: The implementation of an educative program directed to professionals of health in charge of the prenatal control can improve the conditions of the oral health in the pregnant women.
\end{abstract}

Key words: Dentistry, Pregnancy, Oral education, Oral health, Public health.

* Grupo de Investigación CYDUSTA.

Recibido para publicación: 8 de abril de 2010. Aceptado para publicación: 18 de junio de 2010. 


\section{INTRODUCCIÓN}

El embarazo es un estado dinámico y cambiante desde la fecundación hasta el parto. La mujer sufre cambios fisiológicos producidos por las hormonas de la placenta, que además de las modificaciones sistémicas, causan manifestaciones orales, las cuales en su mayoría, desaparecen después del parto. Está demostrado que estas alteraciones pasan inadvertidas por los profesionales encargados del control prenatal, probablemente por la falta de integración entre la consulta médica y la atención odontológica. ${ }^{1-4}$ La mayoría de las mujeres en etapa de gestación no ha recibido instrucciones relacionadas con su salud oral, a pesar de la gran receptividad frente a los cuidados que debe considerar para mantener su salud y la de su bebé; aspecto de suma trascendencia para introducir programas preventivos. ${ }^{5-7}$

El decreto 3039 del 2007 por el cual se adopta el Plan Nacional de Salud Pública 2007- 2010 traza como uno de sus objetivos mejorar la Salud Oral. ${ }^{8}$ Una de las políticas planteadas consiste en fomentar estrategias de información para las embarazadas y de educación al personal de la salud sobre la importancia del componente de salud oral en el control prenatal y su impacto en el control del riesgo de la preeclampsia. Sin embargo, no se halló evidencia de programas educativos desarrollados por odontólogos dirigidos a brindar apoyo al personal responsable del programa de control prenatal, a pesar de la reconocida importancia de la promoción de la salud oral y prevención de la enfermedad en la población de gestantes.

Es así como se confirma la necesidad de implementar acciones efectivas para orientar al personal de salud encargado del control prenatal, con el propósito de que ellos a su vez, brinden información adecuada a las gestantes que acuden a este programa, para mejorar su salud oral y modificar probablemente los perfiles de morbi-mortalidad en el binomio madrehijo; ya que se ha encontrado que la enfermedad periodontal es un factor de riesgo asociado con bajo peso al nacer y parto pretérmino. ${ }^{9-13}$

El propósito de este estudio fue implementar y evaluar un programa educativo dirigido al personal de salud responsable del control prenatal, orientado a promover la salud oral de las gestantes.

\section{MATERIALES Y MÉTODOS}

Se desarrolló un ensayo clínico no controlado donde se intentó comparar la efectividad de un programa educativo ejecutado en dos grupos de gestantes y en dos de profesionales seleccionados por conveniencia. ${ }^{14}$ Para definir el universo se indagó en el Hospital Militar (HOSMIR) de la ciudad de Bucara- manga, el número de profesionales encargados del control prenatal y el número de mujeres embarazadas que acuden mensualmente al dispensario para dicho control. Se obtuvieron los datos de 13 profesionales que dirigían el control prenatal, este universo fue la muestra. De las 80 gestantes de la base de datos, 40 cumplían con los criterios de inclusión.

Para obtener el universo del grupo control se indagó en la Unidad Intermedia Materno Infantil Santa Teresa (UIMIST), el número de profesionales de la salud encargados del control prenatal y el número de mujeres embarazadas que acuden mensualmente. Se adquirieron los datos de 13 profesionales, este universo fue la muestra. De las 120 gestantes registradas en la base de datos, 40 de ellas cumplían con los criterios de inclusión establecidos.

Las variables sociodemográficas, asistencia a la consulta odontológica, nivel de conocimiento sobre enfermedades orales, métodos de higiene oral, mitos sobre la asistencia al odontólogo durante el embarazo, Índice Comunitario Periodontal de Necesidad de Tratamiento, COP, e Índice Cuantificado de Placa se consignaron en un instrumento para las gestantes. Para los profesionales, se consideraron variables sociodemográficas, y nivel de conocimiento en salud oral.

Se realizaron dos instrumentos, uno estaba dirigido a los profesionales y el otro a las gestantes. Se efectuó una prueba piloto previa a la recolección de los datos para evaluar el instrumento, los tiempos requeridos y la comprensión de las preguntas por parte de las gestantes y de los profesionales. Además, las examinadoras fueron estandarizadas para realizar el COP, el Índice Cuantificado de Placa y el Índice Periodontal Comunitario de Necesidad de Tratamiento.

Los datos se sistematizaron en Excel y se exportaron al paquete estadístico EPI-INFO 6.04:15,16 mediante la rutina Validate se verificó la calidad de digitación, finalmente se exportó al paquete STATA 9.1. ${ }^{17} \mathrm{El}$ plan de análisis estadístico univariado implicó el cálculo de medidas de resumen según la naturaleza de las variables; para las cualitativas se calculó proporción, para las cuantitativas medidas de tendencia central, y para la dispersión rangos se usó desviación estándar y varianza.

El plan de análisis bivariado consideró como variable de salida la intervención, y se calculó para las variables cualitativas el test de $\mathrm{Chi}^{2}$ o exacto de Fisher y para las cuantitativas prueba de $t$ de Student o test de rangos de Wilcoxon. ${ }^{18,19}$

La resolución 008430 del Ministerio de Salud de 1993 clasifica este estudio como de riesgo mínimo. ${ }^{20}$ 


\section{RESULTADOS}

\section{Análisis univariado en profesionales}

Se evaluaron 26 profesionales de la salud encargados del control prenatal. La edad promedio fue $36 \pm 6,8$ (13), el 65,4\%(17) era de sexo femenino, el $46,2 \%(12)$ nació en la ciudad de Bucaramanga, el $80,8 \%(21)$ procedía de la zona urbana, el 69,2\%(18) presentaba estudios universitarios y el $26,9 \%(7)$ eran médicos generales (Tabla 1).

Con relación a los conocimientos sobre patologías orales. El personal de la salud presentó un nivel de conocimiento antes de la intervención bueno con un 53,8\% (14) sobre prevención de caries dental y enfermedad periodontal (Tabla 2).

Tabla 1. Descripción de las variables sociodemográficas de los profesionales de la salud encargados del control prenatal analizados en forma global y según intervención.

\begin{tabular}{|c|c|c|c|c|}
\hline Variable & $\begin{array}{c}\text { Global } \\
\mathrm{n}=26 \\
\text { Frec (\%) }\end{array}$ & $\begin{array}{c}\text { Intervenidos } \\
\mathbf{n}=13 \\
\text { Frec (\%) }\end{array}$ & $\begin{array}{c}\text { No Intervenidos } \\
\mathbf{n}=13 \\
\text { Frec (\%) }\end{array}$ & $\mathbf{P}^{*}$ \\
\hline Población & $26(100)$ & $13(50)$ & $13(50)$ & \\
\hline Edad & $36 \pm 6.8$ & $33.6 \pm 6.4$ & $38.4 \pm 6.6$ & $0,0742^{*}$ \\
\hline \multicolumn{5}{|l|}{ Sexo } \\
\hline Mujer & $17(65,4)$ & $9(52,9)$ & $8(47,1)$ & \multirow{2}{*}{$0,680^{* *}$} \\
\hline Hombre & $9(34,6)$ & $4(44,4)$ & $5(55,6)$ & \\
\hline \multicolumn{5}{|l|}{ Lugar de nacimiento } \\
\hline Bucaramanga & $12(46,2)$ & $8(66,7)$ & $4(33,3)$ & \multirow{2}{*}{$0,013^{* *}$} \\
\hline Otro & $14(53,9)$ & $5(35,7)$ & $9(64,3)$ & \\
\hline \multicolumn{5}{|l|}{ Procedencia } \\
\hline Urbana & $21(80,8)$ & $13(61,9)$ & $8(38,1)$ & \multirow{2}{*}{$0,013^{* *}$} \\
\hline Rural & $5(19,2)$ & --- & $5(100)$ & \\
\hline \multicolumn{5}{|l|}{ Educación } \\
\hline Técnico & $3(11,5)$ & $1(33,3)$ & $2(66,7)$ & \multirow{4}{*}{$0,685^{* *}$} \\
\hline Universitario & $18(69,2)$ & $10(55,6)$ & $8(44,4)$ & \\
\hline Especialización & $5(19,2)$ & $2(40)$ & $3(60)$ & \\
\hline Maestría & ---- & ---- & ---- & \\
\hline \multicolumn{5}{|l|}{ Cargo institucional } \\
\hline Medico general & $7(26,9)$ & $4(57,1)$ & $3(42,3)$ & \multirow{7}{*}{$0,985^{* *}$} \\
\hline Odontólogo & $4(15,4)$ & $2(50)$ & $2(50)$ & \\
\hline Nutricionista & $3(11,5)$ & $2(66,7)$ & $1(33,3)$ & \\
\hline Enfermero(a) jefe & $3(11,5)$ & $1(33,3)$ & $2(66,7)$ & \\
\hline Psicólogo & $2(7,7)$ & $1(50)$ & $1(50)$ & \\
\hline Auxiliar enfermería & $2(7,7)$ & $1(50)$ & $1(50)$ & \\
\hline Ginecólogo & $5(19,2)$ & $2(40)$ & $3(60)$ & \\
\hline
\end{tabular}

* $\mathrm{t}$ de Student

${ }^{* *}$ Test exacto de Fisher

$\alpha=0,05$

Tabla 2. Descripción del consolidado de conocimientos sobre patologías orales antes y después en los profesionales de la salud encargados del control prenatal analizados en forma global y según intervención.

\begin{tabular}{|c|c|c|c|c|c|c|c|c|}
\hline \multirow[b]{2}{*}{ Variable } & \multirow{2}{*}{$\begin{array}{l}\text { Global } \\
\text { Antes }\end{array}$} & \multicolumn{2}{|c|}{ Antes } & \multirow[b]{2}{*}{$\mathbf{p}^{*}$} & \multirow{2}{*}{$\begin{array}{l}\text { Global } \\
\text { Después }\end{array}$} & \multicolumn{2}{|c|}{ Después } & \multirow[b]{2}{*}{$\mathbf{p}^{*}$} \\
\hline & & Intervenidos & $\begin{array}{c}\text { No } \\
\text { Intervenidos } \\
\end{array}$ & & & Intervenidos & $\begin{array}{c}\text { No } \\
\text { Intervenidos }\end{array}$ & \\
\hline $\begin{array}{l}\text { Consolidado } \\
\text { conocimiento } \\
\text { en forma } \\
\text { cuantitativa }\end{array}$ & $2,8 \pm 1.5$ & $2,5 \pm 1,7$ & $3,2 \pm 1,3$ & $0,2016^{*}$ & $3,7 \pm 1,2$ & $4 \pm 0,81$ & $3,3 \pm 1,7$ & $0,1555^{*}$ \\
\hline \multicolumn{9}{|c|}{$\begin{array}{l}\text { Consolidado de } \\
\text { conocimientos } \\
\text { en forma } \\
\text { cualitativa }\end{array}$} \\
\hline Bueno & $14(53,8)$ & $6(42,9)$ & $8(57,1)$ & $0,431^{* *}$ & $22(84,6)$ & $13(57,1)$ & $9(40,9)$ & $0,030^{m}$ \\
\hline Malo & $12(46,2)$ & $7(58,3)$ & $5(41,7)$ & & $4(15,4)$ & ----- & $4(100)$ & \\
\hline
\end{tabular}




\section{Análisis bivariado en profesionales}

Con relación a los conocimientos sobre patologías orales, se observaron diferencias estadísticamente significativas entre los grupos $(p=0,030)$, porque el porcentaje fue $53,8 \%$ (14) de respuestas acertadas antes de la intervención, que se incrementó después de la intervención a 84,6\% (22) (Tabla 2).

\section{Análisis univariado gestantes}

Se evaluaron 80 gestantes con una edad promedio de $26,1 \pm 7,5(40)$. El 100\% (80) se encontraba en el segundo trimestre de embarazo. Con relación a los conocimientos sobre prevención de caries dental y enfermedad periodontal, presentaron un nivel de conocimiento malo antes la intervención con un $63,8 \%$ (51) (Tabla 3).
Con respecto a las condiciones de salud oral de las gestantes, al inicio del estudio, se observó 18,7 $\pm 5,3$ dientes sanos, 2,5 $\pm 3,3$ cariados, 4,1 $\pm 3,3$ obturados, y 2,7 $\pm 3,8$ perdidos; el COP fue de 9,3 \pm 5,3 (Tabla 4).

Según el estado de salud periodontal y necesidades de tratamiento, el $35 \%$ (28) de las gestantes presentó sangrado, el 32,5\% (26) presentó cálculos, el $23,7 \%$ (19) bolsas de 4 a $5 \mathrm{~mm}$ y el 7,5\% (6) bolsas de $6 \mathrm{~mm}$. Por lo anterior, el 35\% (28) requirió instrucciones de higiene oral, el 56,2\% (45) detartraje, profilaxis e instrucciones de higiene oral, y el 7,5\% (6) tratamiento complejo (Tabla 5).

El índice cuantificado de placa fue regular para el $56,3 \%(45)$ de las gestantes antes de la intervención (Tabla 6).

Tabla 3. Descripción del consolidado de conocimientos sobre prevención oral en las gestantes analizadas en forma global y según intervención.

\begin{tabular}{|c|c|c|c|c|}
\hline Variable & $\begin{array}{c}\text { Global } \\
\mathrm{n}=\mathbf{8 0} \\
\text { frec }(\%)\end{array}$ & $\begin{array}{c}\text { Intervenidas } \\
\mathrm{n}=40 \\
\text { frec }(\%)\end{array}$ & $\begin{array}{c}\text { No intervenidas } \\
\mathrm{n}=\mathbf{4 0} \\
\text { frec }(\%)\end{array}$ & $\mathbf{P}^{*}$ \\
\hline $\begin{array}{l}\text { Consolidad de conocimientos } \\
\text { en forma cuantitativa }\end{array}$ & $3,9 \pm 1,5$ & $3,9 \pm 1,5$ & $3,9 \pm 1,5$ & $0,8850^{*}$ \\
\hline $\begin{array}{l}\text { Consolidado de } \\
\text { conocimientos en forma } \\
\text { cualitativa } \\
\text { Bueno } \\
\text { Regular } \\
\text { Malo }\end{array}$ & $\begin{array}{c}-\overline{(36,2)} \\
51(63,8)\end{array}$ & $\begin{array}{c}----- \\
15(51,7) \\
25(49,0)\end{array}$ & $\begin{array}{c}---- \\
14(48,3) \\
26(51)\end{array}$ & $0,816^{* *}$ \\
\hline
\end{tabular}

Tabla 4. Descripción de las condiciones de salud oral de las gestantes analizadas en forma global antes y después de la intervención.

\begin{tabular}{|c|c|c|c|c|c|c|c|c|}
\hline \multirow[b]{2}{*}{ Variable } & \multicolumn{4}{|c|}{ Antes } & \multicolumn{4}{|c|}{ Después } \\
\hline & $\begin{array}{l}\text { Global } \\
\text { Antes }\end{array}$ & Intervenidas & $\begin{array}{c}\text { No } \\
\text { Intervenidas }\end{array}$ & $\mathbf{P}^{*}$ & $\begin{array}{c}\text { Global } \\
\text { Después }\end{array}$ & Intervenidas & $\begin{array}{c}\text { No } \\
\text { intervenidas }\end{array}$ & $\mathbf{P}^{*}$ \\
\hline Sanos & $18,7 \pm 5,3$ & $19,9 \pm 4,9$ & $17,5 \pm 5,4$ & $0,5230^{*}$ & $18,7 \pm 5,3$ & $19,9 \pm 4,9$ & $17,5 \pm 5,4$ & $0,0342^{* *}$ \\
\hline Cariados & $2,5 \pm 3,3$ & $2,9 \pm 3,3$ & $2,1 \pm 3,3$ & $0.2540^{* *}$ & $2 \pm 2,7$ & $2,1 \pm 2,6$ & $1,9 \pm 2,9$ & $0,8082^{* *}$ \\
\hline Obturados & $4,1 \pm 3,3$ & $3,1 \pm 3,2$ & $5,1 \pm 3,3$ & $0,0067^{* *}$ & $4,6 \pm 3,3$ & $4 \pm 3,2$ & $5,3 \pm 3,2$ & $0,0735^{* *}$ \\
\hline Perdidos & $2,7 \pm 3,8$ & $2,1 \pm 3,2$ & $3,4 \pm 4,2$ & $0,1182^{* *}$ & $2,7 \pm 3,8$ & $2,1 \pm 3,2$ & $3,4 \pm 4,2$ & $0,1182^{* *}$ \\
\hline $\mathrm{COP}$ & $9,3 \pm 5,3$ & $8,1 \pm 4,9$ & $10,6 \pm 5,4$ & $0,0342^{* *}$ & $9,3 \pm 5,2$ & $8,1 \pm 4,9$ & $10,6 \pm 5,4$ & $0,0342^{* *}$ \\
\hline
\end{tabular}


ARTÍCULO ORIGINAL

Tabla 5. Descripción del estado periodontal y las necesidades de tratamiento de las gestantes, analizadas en forma global antes y después de la intervención.

\begin{tabular}{|c|c|c|c|c|c|c|c|c|}
\hline \multirow[b]{2}{*}{ Variable } & \multirow[b]{2}{*}{$\begin{array}{l}\text { Global } \\
\text { Antes }\end{array}$} & \multicolumn{2}{|c|}{ Antes } & \multirow[b]{2}{*}{$\mathbf{P}^{*}$} & \multirow[b]{2}{*}{$\begin{array}{l}\text { Global } \\
\text { Después }\end{array}$} & \multicolumn{2}{|c|}{ Después } & \multirow[b]{2}{*}{$\mathbf{P}^{*}$} \\
\hline & & Intervenidas & $\begin{array}{c}\text { No } \\
\text { Intervenidas } \\
\end{array}$ & & & Intervenidas & $\begin{array}{c}\text { No } \\
\text { Intervenidas } \\
\end{array}$ & \\
\hline \multicolumn{9}{|l|}{$\begin{array}{l}\text { Índice periodontal } \\
\text { comunitario }\end{array}$} \\
\hline Normal & $1(1,3)$ & --- & $1(100)$ & $0,153^{*}$ & $17(21,3)$ & $16(94,1)$ & $1(5,9)$ & $0,000^{*}$ \\
\hline Sangrado & $28(35)$ & $18(64,3)$ & $10(35,7)$ & & $30(37,5)$ & $20(66,7)$ & $10(33,3)$ & \\
\hline Cálculo & $26(32,5)$ & $14(53,8)$ & $12(46,2)$ & & $12(15)$ & --- & $12(100)$ & \\
\hline Bolsa de 4 a $5 \mathrm{~mm}$ & $19(23,7)$ & $6(31,6)$ & $13(68,4)$ & & $17(21,3 \%)$ & $4(23,5 \%)$ & $13(76,5 \%)$ & \\
\hline Bolsa de $6 \mathrm{~mm}$ & $6(7,5)$ & $2(33,3)$ & $4(66,7)$ & & $4(5 \%)$ & --- & $4(100 \%)$ & \\
\hline \multicolumn{9}{|l|}{$\begin{array}{l}\text { Necesidad de } \\
\text { tratamiento }\end{array}$} \\
\hline No requiere & $1(1,3)$ & --- & $1(100)$ & $0,153^{*}$ & $17(21,3)$ & $16(94,1)$ & $1(5,9)$ & $0,000^{*}$ \\
\hline $\begin{array}{l}\text { Instrucciones de } \\
\text { higiene oral }\end{array}$ & $28(35)$ & $18(64,3)$ & $10(35,7)$ & & $30(37,5)$ & $20(66,7)$ & $10(33,3)$ & \\
\hline $\begin{array}{l}\text { Detartraje, } \\
\text { profilaxis e } \\
\text { instrucciones de } \\
\text { higiene oral }\end{array}$ & $26(32,5)$ & $14(53,8)$ & $12(46,2)$ & & $12(15)$ & --- & $12(100)$ & \\
\hline $\begin{array}{l}\text { Detartraje, } \\
\text { profilaxis e } \\
\text { instrucciones de } \\
\text { higiene oral }\end{array}$ & $19(23,7)$ & $6(31,6)$ & $13(68,4)$ & & $17(21,3)$ & $4(23,5)$ & $13(76,5)$ & \\
\hline $\begin{array}{l}\text { Detartraje, } \\
\text { profilaxis e } \\
\text { instrucciones } \\
\text { de higiene oral } \\
\text { + tratamiento } \\
\text { complejo }\end{array}$ & $6(7,5)$ & $2(33,3)$ & $4(66,7)$ & & $4(5)$ & --- & $4(100)$ & \\
\hline
\end{tabular}

*Test exacto de Fisher $\alpha=0,05$

Tabla 6. Descripción del índice cuantificado de placa de las gestantes, analizadas en forma global antes y después de la intervención.

\begin{tabular}{|c|c|c|c|c|c|c|c|c|}
\hline \multirow{2}{*}{ Variable } & \multirow{2}{*}{$\begin{array}{l}\text { Global } \\
\text { Antes }\end{array}$} & \multicolumn{2}{|c|}{ Antes } & \multirow[b]{2}{*}{$\mathbf{P}^{*}$} & \multirow{2}{*}{$\begin{array}{c}\text { Global } \\
\text { Después }\end{array}$} & \multicolumn{2}{|c|}{ Después } & \multirow[b]{2}{*}{$\mathbf{P}^{*}$} \\
\hline & & Intervenidas & $\begin{array}{c}\text { No } \\
\text { Intervenidas }\end{array}$ & & & Intervenidas & $\begin{array}{c}\text { No } \\
\text { Intervenidas }\end{array}$ & \\
\hline $\begin{array}{l}\text { Índice } \\
\text { cuantificado } \\
\text { de placa } \\
\text { en forma } \\
\text { cuantitativa }\end{array}$ & $0,8 \pm 0,6$ & $0,9 \pm 0,6$ & $0,8 \pm 0,7$ & $0,3845^{*}$ & $1 \pm 0,5$ & $0,8 \pm 0,4$ & $1,1 \pm 0,5$ & $0,0016^{*}$ \\
\hline $\begin{array}{l}\text { Índice } \\
\text { cuantificado } \\
\text { de placa } \\
\text { en forma } \\
\text { cualitativa }\end{array}$ & & & & & & & & \\
\hline Bueno & $25(31,3)$ & $10(40)$ & $15(60)$ & $0,459^{*}$ & $40(50)$ & $25(62,5)$ & $15(37,5 \%)$ & $0,072^{* *}$ \\
\hline Regular & $45(56,3)$ & $25(55,6)$ & $20(44,4)$ & & $33(41,3)$ & $13(39,4)$ & $20(60,6 \%)$ & \\
\hline Malo & $10(12,5)$ & $5(50)$ & $5(50)$ & & $7(8,7)$ & $2(28,6)$ & $5(71,4 \%)$ & \\
\hline
\end{tabular}

\section{Análisis bivariado gestantes}

El nivel de conocimiento fue malo en un $49 \%$ (25) en el grupo intervenido y un $51 \%$ (26) en el grupo no intervenido (Tabla 3).

Las condiciones de salud oral se vieron afectadas antes y después de la intervención; se observaron dife- 
rencias estadísticamente significativas $(p=0,0342)$ en la variable COP con un promedio de $8,1 \pm 4,9$ en el grupo intervenido y 10,6 \pm 5,4 para el grupo no intervenido (Tabla 4). En lo referente al estado de salud periodontal y necesidad de tratamiento, se observó la tendencia a mejoría en la salud oral del grupo intervenido mientras en el grupo control o no intervenido, no se apreciaron cambios (Tabla 5).

La misma tendencia se observó para las variables Índice Cuantificado de Placa y Necesidades de Tratamiento en el grupo intervenido. En el grupo no intervenido estas variables se comportaron igual al inicio y al final del estudio (Tabla 6).

\section{DISCUSIÓN}

Se reconoce que el programa educativo que se implementó no fue asociado directamente al aprendizaje evaluado por medio de los instrumentos; aunque se observó en los resultados un cambio de conocimientos que pudieron haber mejorado por otros factores de índole informativo.

Los profesionales de la salud que dirigen los programas de control prenatal son los encargados de transmitir información sobre todos los cambios, riesgos y prevención durante el periodo de gestación. ${ }^{21-24}$ Sin embargo, los temas relacionados con la salud oral está excluido de estos programas.

En el decreto 3039 en el objetivo 3 se habla de mejorar la salud oral y en sus estrategias de línea política 1,2 y 3 plantea fomentar una estrategia de información a las embarazadas y de educación al personal de salud sobre la importancia del componente de salud oral en el control prenatal y su impacto en el riesgo de la preeclampsia., ${ }^{8,24}$ De esta propuesta surge la necesidad de intervenir a los profesionales de la salud encargados del control prenatal mediante un programa educativo diseñado para informar sobre la importancia del componente de promover la salud oral como parte de la integralidad de la embarazada.

Al iniciar el estudio se observó que el conocimiento de los profesionales de la salud sobre la prevención oral en las gestantes fue de $42,9 \%$ antes de la intervención mejorando a través del programa educativo a un $57,1 \%$.

El resultado en cuanto al conocimiento de las gestantes en el presente estudio fue entre regular y malo, este resultado se encontró similar al estudio de Sierra y colaboradores que observaron un nivel de conocimiento insatisfactorio. ${ }^{26}$ Esta condición refleja la necesidad de interacción del cuerpo odontológico con el grupo de control prenatal y las gestantes para mejorar el nivel de salud oral y el interés en las condiciones de salud oral. ${ }^{1,2,27}$
En cuanto al índice de COP el promedio que se encontró en las gestantes fue del 9,3, similar al índice encontrado por Riveros y Rodríguez con un de COPd de 9.4 al inicio del estudio. ${ }^{1}$ Coincide también con el estudio de Carrión donde su promedio de COPd fue de $8,26{ }^{28}$ Se concluye que la gestación es un tiempo relativamente corto para que el número de caries dental se incremente significativamente, y que a pesar de las condiciones implícitas que éste conlleva, no es una patología que necesariamente se agrave con el embarazo.

En el estudio realizado por Carrión se observó un $100 \%$ de algún tipo de enfermedad periodontal con alta presencia de sangrado y cálculo, además de una alta presencia de bolsas entre 4 y $5 \mathrm{~mm}$ en el maxilar superior relacionándose con la formación de seudobolsas producto de la inflamación gingival que se produce en el embarazo por los cambios hormonales. ${ }^{28}$ El presente estudio confirmó que el $98.7 \%$ presentaban algún tipo de alteración en su periodonto. Los resultados reportados por Chacón y colaboradores mostraron un $2,68 \%$ de las gestantes con periodonto sano mientras que Riveros y Rodríguez no reportaron ninguna mujer periodontalmente sana. ${ }^{1,2}$

La resolución 00412 del 2000 define en su artículo quinto a la "Demanda Inducida", como el conjunto de acciones encaminadas a informar y educar a la población, para dar cumplimiento a las actividades e intervenciones de protección específica y detección temprana establecidas en las normas técnicas, pero no se encuentran acciones directas para intervenir dicha población..$^{29}$ Según Saldarriaga y colaboradores se reporta que la atención estomatológica en la gestante es un problema que debe interesar no sólo a los odontólogos sino también al personal médico que en su práctica se relaciona con estos grupos de la población. ${ }^{23,30}$ Es de gran importancia que el personal médico tenga los conocimientos necesarios, con el fin de que pueda no sólo hacer prevención, sino también detectar las enfermedades orales más frecuentes y de esta forma orientar y remitir con mayor frecuencia al paciente al odontólogo y crear así una demanda a la consulta odontológica. ${ }^{23,30}$

Estos hallazgos confirman la receptividad de los grupos estudiados a la intervención porque más gestantes en control prenatal fueron remitidas por su médico a odontología y se aumentaron los niveles de salud oral.

\section{Conclusiones}

La efectividad de un programa educativo dirigido a profesionales de la salud encargados del control prenatal puede mejorar las condiciones de salud oral en las gestantes. Si se crea un canal de comuni- 
cación más estrecho y se consigue la participación activa de las gestantes y profesionales involucrados en los programas de control prenatal.

Es imperiosa la necesidad de implementar capacitaciones y evaluaciones de conocimientos de los profesionales de salud sobre la promoción de la salud oral y la prevención como componente primordial del control prenatal.

\section{BIBLIOGRAFÍA}

1. Riveros CM, Rodríguez MJ. Programa preventivo en salud oral para controlar factores de riesgo asociados a caries dental y enfermedad periodontal en mujeres gestantes. Ustasalud 2006; 5: 17 - 28.

2. Chacón AF, Gutiérrez ZA, Montañez J, Pieschacón MP, Camargo DM. Factores asociados a caries y enfermedad periodontal en mujeres gestantes. Ustasalud 2002; 1: 33 - 39.

3. Marrero A, López E, Sayas SC, Ribacoba E. Diagnostico educativo en salud bucal para gestantes. Archivo médico Camagüey 2004; 8.

4. Zanata RL, Navarro MF, Pereira JC, Franco EB, Lauris JR, Barbosa SH. Effect of caries prevents measures direct to expectant mother on caries experience in their children. Braz Dent J 2003; 14: 75 - 81.

5. Carozo BJ, Miqueri AG, Esquivel CV, Vera MA, Larrosa ES. Prevención en embarazadas: un doble beneficio [en línea]. Universidad Nacional del Nordeste. Comunicaciones Científicas y Tecnológicas 2006 [Fecha de acceso: mayo de 2009]. URL disponible en: http://www.unne. edu.ar/Web/cyt/cyt2006/03-Medicas/2006-M 119.pdf

6. Olivar A. Cambios biológicos, psicológicos y sociales durante el embarazo. Revista Federación Odontológica Colombiana 2001; 201: $37-51$.

7. Gonzaga HF, Buso L, Jorge MA, Gonzaga LH. Intrauterine Dentistry: An Integrated Model of Prevention. Braz Dent J 2001; 12: 139 - 142.

8. República de Colombia. Ministerio de Salud. Decreto 3039 del 2007, Agosto 10, por el cual se expide los Objetivos de las Prioridades Nacionales en Salud para el periodo 2007 -2010 Objetivo 3.

9. Maita LV, Maita LM. La enfermedad periodontal como factor de riesgo para nacimiento pretérmino. Odontología Sanmarquina 2003; 6: 37 - 40.

10. Vettore MV, Lamarca GA, Leâo AT, Thomaz FB, Sheiham A, Leal MC. Periodontal infection and adverse pregnancy outcomes: a systematic review of epidemiological studies. Cad Saúde Pública, Rio de Janeiro 2006; 22: 2041 2053.

11. Michalowicz BS, Hodges JS, DiAngelis AJ, Lupo VR, Novak J, Ferguson JE, et al. Treatment of periodontal disease and the risk of preterm birth. N Engl J Med 2006; 355: 1885 -1894 .

12. Offenbacher S, Boggess KA, Murtha AP,Heather L. Jared, MS, Lieff S, McKaig RG, Mauriello SM, Moss KL, Beck JD. Progressive periodontal disease and risk of very preterm delivery. Obstet Gynecol 2006; 107: 29 - 36.

13. Boggess KA, Edelstein BL. Oral health in women during preconception and pregnancy: implications for birth outcomes and infant oral health. Matern Child Health J 2006; ; 10: S169 - S174.

14. Cunningham SJ. How to...write a paper. J Orthod 2004; 31: $47-51$.
15. Microsoft Office Excel 5.0, Microsoft Corporation 2007.

16. CDC-OMS. Epi-inf 6 Versión 6.04 ${ }^{\mathrm{a}}$ Nov 1996.

17. Stata corp 2003. Stata Statistical Software. Release 9.1 College Station, T.X: Stata Corporation.

18. Pagano M, Gauvreau K, Principles of Biostatistics. Duxbury Press. Belmont p. 35 - 44, 257 -263.

19. Norman GR, Streiner DL. Bioestadística. Mosby/ Doyma Libros. Madrid. 1996 p 129 - 147.

20. República de Colombia. Ministerio de Salud. Resolución número 008430 de 1993, 4 de octubre, por la cual se establecen las normas científicas, técnicas y administrativas para la investigación en salud.

21. Elías MC. Importancia de la educación como sostén de la prevención de caries dental. Odontopediatria y Prevención en salud bucal 2003; 1-5. [en línea]. URL disponible en: http://www.geodental.net/modules.php?name $=$ Rec ursos\&mop $=$ article\&recid $=6916$

22. Torralba G. La relación de la paciente con el odontólogo. [en línea] BGYN.netlatina. [fecha de acceso: junio de 2010].

23. Saldarriaga OJ, Sánchez M, Avendaño L. Conocimientos y prácticas en salud bucal de las gestantes vinculadas al programa de control prenatal. CES Odontología 2003; 17: 9 - 23.

24. Cancino E, León H, Pérez González E, Sarmiento R, Bosa A. Guía de control prenatal y factores de riesgo [en línea]. Secretaria Distrital de Salud de Bogotá, Asociación Bogotana de Ginecología y Obstetricia [Fecha de acceso: mayo de 2009]. 1 - 39. URL disponible en: http://www. saludcapital.gov.co/Publicaciones/Desarrollo\%20de\%20 Servicios/Gu\%C3\%ADas\%20para\%20la\%20atención\%20 Materno\%20Perinatal/GUIA\%201.\%20\%20CONTROL\%20 PRENATAL\%20Y\%20FACTORES\%20DE\%20RIESGO.pdf

25. Castaldi JL, Bertin MS, Jiménez F,llld R. Enfermedad periodontal ¿Es factor de riesgo para parto pretermino, bajo peso al nacer o preeclampsia? Rev Panam Salud Pública 2006; 19: 253 - 258.

26. Almarales C, Llerandi Y. Conocimientos sobre salud bucal y demanda de servicios estomatológicos en relación con la enfermedad periodontal en embarazadas. Rev Cubana Estomatol [en línea]. 2008 [fecha de acceso: junio de 2009]; 45 (2). URL disponible en: http:// scielo.sld.cu/scielo.php?script $=$ sci_arttext\&pid $=$ S0034$75072008000200003 \& \operatorname{lng}=$ es.

27. Iftime C, Galuscan A, Jumanca D, Podariu A, Oanca R, Popovic R, Sava- Rosianu R. Health education and prophylactic measures need during pregnancy. Medicine in Evolution 2008; 1: 117 - 124.

28. Carrión C. Diagnóstico de salud oral de primigestas que acuden a control prenatal al consultorio Dr. José D. Asta Buruanga [Trabajo de Grado] Talca: Universidad de Talca. Facultad de Ciencias de la Salud; 2004. Pág. 1 - 71.

29. República de Colombia. Ministerio de Salud. Resolución número 00412 de 2000 , febrero 25 , por el cual se establecen las actividades, procedimientos, e intervenciones de la demanda inducida y obligatorio cumplimiento y se adoptan las normas técnicas y guías de atención para el desarrollo de las acciones de protección específica y detección temprana y la atención de enfermedades de interés en salud pública.

30. Saldarriaga CA, Saldarriaga POJ. El médico general y el pediatra en la promoción de la salud oral y la prevención de la enfermedad del niño menor de cinco años y la mujer en período de gestación. CES Odontología 2002; 15 : $13-20$. 